\title{
SMOOTH PRIME FANO COMPLETE INTERSECTIONS IN TORIC VARIETIES
}

\author{
VICTOR PRZYJALKOWSKI AND CONSTANTIN SHRAMOV
}

\begin{abstract}
We prove that a smooth well formed Picard rank one Fano complete intersection of dimension at least 2 in a toric variety is a weighted complete intersection.
\end{abstract}

An obvious way to construct new Fano varieties is to describe them as divisors or complete intersections of divisors in those varieties that are already known and well understood: for instance, in toric varieties (in particular, in weighted projective spaces), or in Grassmannians. Fano varieties that are complete intersections in Grassmannians were studied by several authors, see, for instance, [Kü95, [Kü97, or [FM18]. As for Fano weighted complete intersections, they also attracted some attention, see e.g. [CCC11, [PST17], [PSh19], [PSh20a], [PSh20b], PSh20c]. The most interesting case from the point of view of classification is the case of prime Fano varieties, that is ones of Picard rank one. Note that this property automatically holds for smooth complete intersections (of dimension at least 3) in Grassmannians or weighted projective spaces by the Lefschetz theorem. On the other hand, for complete intersections in other toric varieties it does not hold often. In this note we prove that the only prime Fano complete intersections of dimension at least 2 in toric varieties are weighted complete intersections.

First we remind the classical construction of a toric variety as a quotient (see [Co95]). Let $Y$ be a $\mathbb{Q}$-factorial projective toric variety; here and below all varieties are assumed to be defined over the field $\mathbb{C}$ of complex numbers. Let $D_{1}, \ldots, D_{b}$ be its prime boundary divisors. They generate the divisor class group $\mathrm{Cl}(Y)$. Consider the $\mathrm{Cl}(Y)$-graded algebra $S=\mathbb{C}\left[x_{1}, \ldots, x_{b}\right]$ with grading defined by

$$
\operatorname{deg}\left(\prod_{i=1}^{b} x_{i}^{r_{i}}\right)=\sum_{i=1}^{b} r_{i} D_{i} .
$$

One has $\operatorname{Spec}(S) \cong \mathbb{A}^{b}$, and there is a natural correspondence between rays $e_{i}$ of a fan of $Y$ and variables $x_{i}$. Define the subvariety $Z$ in $\operatorname{Spec}(S)$ as the intersection of hypersurfaces $\left\{\prod x_{i}=0 \mid e_{i} \notin \sigma\right\}$ over all cones $\sigma$ of a fan of $Y$. Then $Y$ is a geometric quotient of

$$
U=\operatorname{Spec}(S) \backslash Z \subset \mathbb{A}^{b}
$$

by the abelian linear algebraic group

$$
\mathbf{D}=\operatorname{Hom}_{\mathbb{Z}}\left(\mathrm{Cl}(Y), \mathbb{C}^{*}\right) .
$$

Example 1. Let $Y=\mathbb{P}\left(a_{0}, \ldots, a_{N}\right)$ be a weighted projective space of dimension $N$. Suppose that the greatest common divisor of the numbers $a_{0}, \ldots, a_{N}$ equals 1 (which can be always achieved by cancelling the greatest common divisor of $\left.a_{0}, \ldots, a_{N}\right)$. Recall that nevertheless

Victor Przyjalkowski was supported by President Grant MD-30.2020.1. Constantin Shramov was supported by the HSE University Basic Research Program, Russian Academic Excellence Project "5-100". Both authors are supported by the Foundation for the Advancement of Theoretical Physics and Mathematics "BASIS". 
the collection $a_{0}, \ldots, a_{N}$ is not uniquely defined by $Y$. However for a suitable choice of the weights $a_{0}, \ldots, a_{N}$ the fan of the toric variety $Y$ in $\mathbb{Z}^{N}$ has $N+1$ rays with primitive vectors $v_{0}, \ldots, v_{N}$ subject to the relation

$$
a_{0} v_{0}+\ldots+a_{N} v_{N}=0
$$

see [Do82, 1.2.5]. Using the description of $\mathrm{Cl}(Y)$ via the rays of the fan, we see that $\mathrm{Cl}(Y) \cong \mathbb{Z}$. This means that $\mathbf{D} \cong \mathbb{C}^{*}$. Furthermore, one has $Z=\{0\} \subset \mathbb{A}^{N+1}$, so that

$$
Y \cong\left(\mathbb{A}^{N+1} \backslash\{0\}\right) / \mathbb{C}^{*}
$$

Example 2 ([Kas09, Example 1.3]). Let $Y$ be a three-dimensional toric variety whose fan has rays generated by the vectors

$$
(1,0,0), \quad(0,1,0), \quad(1,-3,5), \quad(-2,2,-5)
$$

in $\mathbb{Z}^{3}$. One can see that

$$
\mathrm{Cl}(Y) \cong \mathbb{Z} \oplus \mathbb{Z} / 5 \mathbb{Z}
$$

Therefore, we have

$$
\mathbf{D}=\operatorname{Hom}_{\mathbb{Z}}\left(\mathbb{Z} \oplus \mathbb{Z} / 5 \mathbb{Z}, \mathbb{C}^{*}\right) \cong \mathbb{C}^{*} \times \mathbb{Z} / 5 \mathbb{Z} .
$$

In particular, $\mathbf{D}$ is not connected in this case. One has

$$
Y \cong\left(\mathbb{A}^{4} \backslash\{0\}\right) / \mathbf{D} \cong \mathbb{P}^{3} /(\mathbb{Z} / 5 \mathbb{Z}) .
$$

Remark 1. According to [BB93, the threefold $Y$ is the unique three-dimensional toric variety with terminal singularities that has Picard rank 1 and is not a weighted projective space.

Example 3. Let $Y$ be a four-dimensional toric variety whose fan has rays generated by the vectors

$$
(1,0,0,0), \quad(0,1,0,-1), \quad(0,0,-1,0), \quad(0,0,2,-1), \quad(-1,-1,-1,2)
$$

in $\mathcal{N} \cong \mathbb{Z}^{4}$. The sum of these vectors is equal to 0 , so $Y$ is a quotient of $\mathbb{P}^{4}$. In fact, one has

$$
Y \cong\left(\mathbb{A}^{4} \backslash\{0\}\right) /\left(\mathbb{C}^{*} \times \mathbb{Z} / 3 \mathbb{Z}\right) \cong \mathbb{P}^{4} /(\mathbb{Z} / 3 \mathbb{Z})
$$

Since any three of the above five vectors form a part of a basis of $\mathcal{N}$, we see that the singularities of $Y$ are isolated.

Example 4 (cf. [Kas09, Example 1.2]). Let $p$ be a positive integer, and let $G=\mathbb{Z} / p \mathbb{Z}$. Consider the action of $G$ on the projective space $\mathbb{P}^{p-1}$ with homogeneous coordinates $x_{0}, \ldots, x_{p-1}$ given by the formula $x_{i} \mapsto \varepsilon^{i} x_{i}$, where $\varepsilon$ is a primitive $p$-th root of unity. Set $Y=\mathbb{P}^{p-1} / G$. Then $Y$ is a $\mathbb{Q}$-factorial toric variety, and

$$
Y \cong\left(\mathbb{A}^{p} \backslash\{0\}\right) /\left(\mathbb{C}^{*} \times \mathbb{Z} / p \mathbb{Z}\right)
$$

Note that if $p$ is a prime number, then the singularities of $Y$ are isolated.

The above examples motivate the following definition.

Definition 1 (cf. [Kas09, Definition 1.1]). An $N$-dimensional toric variety whose fan has $N+1$ rays is called a generalized weighted projective space.

Lemma 1 ([Kas09, Corollary 2.3]). Every generalized weighted projective space is either a weighted projective space, or a quotient of a weighted projective space by a non-trivial finite group acting freely in codimension 1. 
We call a polynomial $f \in S$ homogeneous if for some $d \in \mathrm{Cl}(Y)$ all the monomials of $f$ are of degree $d$. For any homogeneous polynomials $f_{1}, \ldots, f_{k}$ the intersection $C_{X}^{*}$ of their common zero locus with $U$ is stable under the action of $\mathbf{D}$. Thus $C_{X}^{*}$ determines a closed subset $X$ in $Y$.

Note that this description specializes to weighted complete intersections in the case when $Y$ is a weighted projective space. In the same way as for weighted complete intersections we can give standard definitions for the case of complete intersections in toric varieties. Let $X \subset Y$ have codimension $k$, so that $C_{X}^{*} \subset U$ is also of codimension $k$. If there are $k$ generators $f_{1}, \ldots, f_{k}$ of the ideal of $C_{X}^{*}$ in $U$, so that $C_{X}^{*}$ is a complete intersection in $U$, we say that $X$ is a complete intersection of the hypersurfaces that are images in $Y$ of the divisors defined by equations $f_{j}=0$ in $U$. This is equivalent to the regularity of the sequence $f_{1}, \ldots, f_{k}$ in the localization of $S$ with respect to the ideal defining $Z$. We say that $X$ is well formed if

$$
\operatorname{codim}_{X}(X \cap \operatorname{Sing} Y) \geqslant 2 \text {. }
$$

Remark 2. Let $Y$ be a weighted projective space $\mathbb{P}\left(a_{0}, \ldots, a_{N}\right)$ considered as a toric variety. We may assume that the greatest common divisor of the numbers $a_{0}, \ldots, a_{N}$ equals 1 . According to Example 1, for a suitable choice of the weights $a_{0}, \ldots, a_{N}$ the fan of the toric variety $Y$ in $\mathbb{Z}^{N}$ has $N+1$ rays with primitive vectors $v_{0}, \ldots, v_{N}$ subject to relation (1). If all numbers among $a_{0}, \ldots, a_{N}$ except one, say, the numbers $a_{1}, \ldots, a_{N}$, are divisible by some integer $a>1$, then all coordinates of the vector $v_{0}$ are divisible by $a$; the latter is impossible because $v_{0}$ is a primitive vector in $\mathbb{Z}^{N}$. Thus $Y$ is automatically well formed in the sense of [IF00, Definition 5.11]. In other words, the description of $Y$ as a toric variety recovers the unique collection $a_{0}, \ldots, a_{N}$ among all collections of weights defining the same weighted projective space $Y$ such that the weighted projective space is well formed (recall that well formedness in the sense of [IF00] is a property of the weights, not the weighted projective space itself). As a consequence, we conclude that the two notions of well formedness for weighted complete intersections agree with each other.

It appears that many cohomology groups of a complete intersection of ample hypersurfaces in a toric variety $Y$ are defined by the cohomology groups of $Y$, similarly to complete intersections in usual projective spaces.

Theorem 1 (Lefschetz theorem for toric varieties, see [Ma99, Proposition 1.4]). Let $Y$ be a $\mathbb{Q}$-factorial projective toric variety of dimension $N$, and let $X \subset Y$ be a complete intersection of $k$ ample hypersurfaces in $Y$. Then the natural map

$$
H^{i}(Y, \mathbb{Z}) \rightarrow H^{i}(X, \mathbb{Z})
$$

is an isomorphism for $i<N-k=\operatorname{dim}(X)$ and an injection for $i=N-k$.

Corollary 1. A positive-dimensional complete intersection of ample hypersurfaces in a $\mathbb{Q}$ factorial projective toric variety is connected.

Corollary 2. Let $X$ be a complete intersection of ample hypersurfaces in a $\mathbb{Q}$-factorial projective toric variety $Y$. Then the restriction map

$$
\operatorname{Pic}(Y) \rightarrow \operatorname{Pic}(X)
$$

is an isomorphism if $\operatorname{dim}(X) \geqslant 3$, and is injective if $\operatorname{dim}(X)=2$. Furthermore, if $\operatorname{dim}(X) \geqslant 2$ and $X$ is $\mathbb{Q}$-factorial, then $\operatorname{rk} \mathrm{Cl}(X) \geqslant \operatorname{rk} \mathrm{Cl}(Y)$. 
Proof. Note that $H^{1}(Y, \mathbb{Z})=0$, because $Y$ is a toric variety. By Theorem 1, one has $H^{1}(X, \mathbb{Z})=0$; moreover, the map $H^{2}(X, \mathbb{Z}) \rightarrow H^{2}(Y, \mathbb{Z})$ is an isomorphism if $\operatorname{dim}(X) \geqslant 3$, and is injective if $\operatorname{dim}(X)=2$. Thus, the assertions about the Picard group follow from the exponential exact sequence. On the other hand, if $X$ is $\mathbb{Q}$-factorial, one has $\operatorname{rk} \mathrm{Cl}(X)=\operatorname{rk} \operatorname{Pic}(X)$ (and also $\operatorname{rk} \mathrm{Cl}(Y)=\operatorname{rk} \operatorname{Pic}(Y))$.

For the following we need a general fact (see, for instance, [DD85, Corollary 3]).

Proposition 1. Let $U$ and $V$ be normal varieties, and let $f: U \rightarrow V$ be a finite surjective morphism. Then the branch locus $B(f)$ of $f$ has codimension 1 at any point of $B(f) \cap(V \backslash \operatorname{Sing}(V))$.

Proposition 2. Let $X$ be a positive-dimensional well formed Fano complete intersection in $a \mathbb{Q}$-factorial projective toric variety $Y$. Suppose that $Y$ is a quotient of a weighted projective space by a non-trivial finite group $G$ acting freely in codimension 1 . Then $X$ is singular.

Proof. Suppose that $X$ is smooth. Let $\psi: \mathbb{P} \rightarrow Y$ be the quotient map, where $\mathbb{P}$ is a weighted projective space. We claim that the branch locus $B(\psi) \subset Y$ of $\psi$ lies in the singular locus of $Y$. Indeed, by Proposition 1 the locus $B(\psi) \backslash \operatorname{Sing} Y$ has codimension 1 in $Y$. Together with the freeness in codimension 1 of the action of $G$ this implies that

$$
B(\psi) \backslash \operatorname{Sing} Y=\varnothing .
$$

Proposition 1 applied to the covering $X^{\prime} \rightarrow X$, where $X^{\prime}=\psi^{-1}(X)$, implies that this covering is branched in the locus $B(\psi) \cap X$ of codimension 1 in $X$.

Suppose that $B(\psi) \cap X=\varnothing$, so that $X^{\prime} \rightarrow X$ is an unramified covering. Note that $X^{\prime}$ is a complete intersection in $Y$; since $Y$ is a weighted projective space, $X^{\prime}$ is a complete intersection of ample hypersurfaces. Thus it follows from Corollary 1 that $X^{\prime}$ is connected. On the other hand, the smooth Fano variety $X$ has trivial fundamental group (see, for instance, [IP99, Corollary 6.2.18]). The obtained contradiction shows that $B(\psi) \cap X \neq \varnothing$. However, since

$$
B(\psi) \cap X \subset \operatorname{Sing} Y \cap X,
$$

the latter contradicts the well formedness of $X$.

Theorem 2. Let $X$ be a smooth well formed Fano complete intersection of ample hypersurfaces in a $\mathbb{Q}$-factorial projective toric variety $Y . \quad$ Suppose that $\operatorname{dim}(X) \geqslant 2$ and $\operatorname{rk} \operatorname{Pic}(X)=1$. Then $Y$ is a weighted projective space.

Proof. We know from Corollary 2 that $\mathrm{rk} \mathrm{Cl}(Y)=1$. In other words, $Y$ is a generalized weighted projective space. Thus by Lemma 1 the variety $Y$ is either a weighted projective space, or a quotient of a weighted projective space by a non-trivial finite group acting freely in codimension 1. Now the assertion follows from Proposition 2 ,

Note that the assertion of Theorem 2 fails if $X$ has dimension 1. For instance, a smooth rational curve can be embedded into the toric surface $Y=\mathbb{P}^{1} \times \mathbb{P}^{1}$ as an ample hypersurface of bidegree $(1,1)$. Also, Theorem 2 does not hold without the assumption that $X$ is Fano. Indeed, let $Y$ be a generalized weighted projective space of dimension at least 4 with isolated singularities such that $Y$ is not a weighted projective space, see Examples 3 and 4 . Let $X$ be a general divisor from a sufficiently ample linear system on $Y$. Then $X$ is smooth by Bertini theorem, and $\operatorname{rk} \operatorname{Pic}(X)=1$ by Corollary 2. Finally, we point out that the smoothness assumption in Theorem 2 is also essential. Indeed, let $p \geqslant 5$ be a prime number, and let $H$ be the hyperplane defined by the equation $x_{p-1}=0$ in the projective space $\mathbb{P}^{p-1}$ with 
homogeneous coordinates $x_{0}, \ldots, x_{p-1}$. Let $Y$ be the generalized weighted projective space constructed as in Example 4. Then the image of $H$ in $Y$ is a prime Fano hypersurface with terminal singularities.

The authors are grateful to Yu. Prokhorov for his helpful comments.

\section{REFERENCES}

[BB93] A. Borisov, L. Borisov, Singular toric Fano three-folds, Sb. Math., 75:1 (1993), 277-283.

[CCC11] J.-J. Chen, J. Chen, M. Chen, On quasismooth weighted complete intersections, J. Algebraic Geom., 20:2 (2011), 239-262.

[Co95] D. Cox, The homogeneous coordinate ring of a toric variety, J. Algebraic Geom., 4 (1995), 17-50.

[Da78] V.Danilov, The geometry of toric varieties, Russian Math. Surveys, 33:2 (1978), 97-154.

[DD85] A. Dimca, S. Dimiev, On analytic coverings of weighted projective spaces, Bull. London Math. Soc., 17:3 (1985), 234-238.

[Do82] I. Dolgachev, Weighted projective varieties, Lecture Notes in Math., 956 (1982), 34-71. SpringerVerlag, Berlin.

[FM18] E. Fatighenti, G. Mongardi, A note on a Griffiths-type ring for complete intersections in Grassmannians, arXiv:1801.09586 (2018).

[IF00] A. R. Iano-Fletcher, Working with weighted complete intersections, Explicit birational geometry of 3-folds, 101-173, London Math. Soc. Lecture Note Ser., 281, Cambridge Univ. Press, Cambridge, 2000 .

[IP99] V. Iskovskikh, Yu. Prokhorov, Fano varieties, Encyclopaedia of Mathematical Sciences, 47 (1999), Springer, Berlin.

[Kas09] A. Kasprzyk, Bounds on fake weighted projective space, Kodai Math. J., 32:2 (2009), 197-208.

[Kü95] O. Küchle, On Fano 4-fold of index 1 and homogeneous vector bundles over Grassmannians, Math. Z., 218:4 (1995), 563-575.

[Kü97] O. Küchle, Some remarks and problems concerning the geography of Fano 4-folds of index and Picard number one, Quaestiones Math. 20:1 (1997), 45-60.

[Ma99] A. Mavlyutov, Cohomology of complete intersections in toric varieties, Pacific J. Math. 191:1 (1999), 133-144.

[PST17] M.Pizzato, T. Sano, L. Tasin, Effective nonvanishing for Fano weighted complete intersections, Algebra Number Theory, 11:10 (2017), 2369-2395.

[PSh19] V.Przyjalkowski, C.Shramov, Automorphisms of weighted complete intersections, Proc. Steklov Inst. Math., 307 (2019), 198-209.

[PSh20a] V. Przyjalkowski, C. Shramov, Bounds for smooth Fano weighted complete intersections, CNTP, 14:3 (2020), 511-553.

[PSh20b] V. Przyjalkowski, C. Shramov, Fano weighted complete intersections of large codimension, Sib. Math. J., 61:2 (2020), 298-303.

[PSh20c] V.Przyjalkowski, C. Shramov, On automorphisms of quasi-smooth weighted complete intersections, arXiv:2006.01213 (2020).

Victor Przyjalkowski

Steklov Mathematical Institute of RAS, 8 Gubkina street, Moscow 119991, Russia.

victorprz@mi.ras.ru, victorprz@gmail.com

Constantin Shramov

Steklov Mathematical Institute of RAS, 8 Gubkina street, Moscow 119991, Russia.

National Research University Higher School of Economics, Laboratory of Algebraic Geometry, NRU HSE, 6 Usacheva str., Moscow, 117312, Russia.

costya.shramov@gmail.com 